\title{
An enameled wire with a semi-conductive layer: A solution for a better distibution of the voltage stresses in motor windings
}

https://doi.org/10.1515/phys-2019-0067

Received May 29, 2019; accepted Jun 28, 2019

\begin{abstract}
The paper analyzes a possible improvement of Pulse Width Modulation (PWM) fed motor insulation according to a principle developed by the high voltages industry. The proposed principle consists in using a specific enameled wire made with an additional semi-conductive layer on its outer surface.

Experimental study is realized in order to measure Partial Discharges (PDs) activity with three technologies: Classical enameled wire, enameled wire with an external conductive layer (silver paint) and enameled wire with an external semi-conductive layer ( $2 \%$ of carbon nanoparticles). Simulations are made in order to understand the behavior of the new semi-conductive wire.
\end{abstract}

Keywords: Enameled wire, motor winding, partial discharges, semi-conductive layer

PACS: 84.30.Jc, 52.80.-s

\section{Introduction}

Semi-conductive tapes are widely used in connection boxes of high voltage cables [1]. They are also used for getting a correct potential distribution in the end-winding connections of large big machines working in high voltages [2]. These two applications concern macroscopic objects, which sizes are more than 10 centimeters. For getting a better electric fields distribution in critical parts, strict rules must be respected for the position of the additional semi-conductive tape.

\footnotetext{
*Corresponding Author: Sonia Ait-Amar: Univ. Artois, EA4025, LSEE, EA4025, F-62400, Béthune, France; Email: sonia.aitamar@univ-artois.fr Daniel Roger, Emmanuel Mateo: Univ. Artois, EA4025, LSEE, EA4025, F-62400, Béthune, France
}

In a low voltage motor fed by a PWM inverter, similar electric fields exist, but in microscopic areas between the turns of the enameled wire or between the surface of the enameled wire and the magnetic core [3]. The problem is critical for machine working in low-pressure environments, as in aeronautics. The electric fields amplitudes are similar to those of the high voltage industry but not the geometry sizes. Therefore, the electrostatic problems are similar, but at a microscopic scale. The manual methods used for decades in high voltage industry cannot be used but their principles remain valid.

The paper proposes to using an enameled wire made with an additional semi-conductive layer on its external surface for making low-voltage electrical machines fed by PWM inverters. Experimental and simulation approaches are achieved in order to study this proposed solution.

The presented approach is a step toward the design of electrical machine by moving PDs towards critical zones, near the coil connections. Additional small balls of varnish at this area, strongly increase the PDIV of the coil.

\section{Partial discharge detection: experimental setup description}

Electrical machine insulation degradation is linked to Partial Discharges (PDs) that corresponds to the ionization of the air in the vicinity of the enameled wires. During the ionization phenomenon of the air, the charges are deposited on the outer surface of the insulating layer covering the copper wires (Figure 1). This phenomenon is faster than one microsecond; it causes an equivalent current pulse on the external electrical circuit.

For studying the effects of the conductive layer on PDs, measurements have been carried out, according to the IEC 60270 standard [4]. Figure 2 presents the test bench where:

- U: variable $50 \mathrm{~Hz}$ sinusoidal voltage supply;

- LPF: low pass filter reducing noise of power supply;

- Sample: twisted pair under testing;

əopen Access. ๑ 2019 S. Ait-Amar et al., published by De Gruyter. (cc) BY 4.0 License 


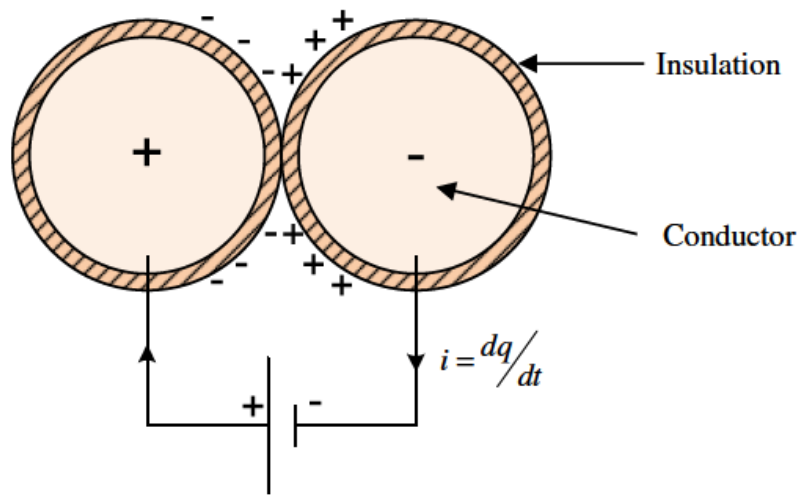

Figure 1: Diagram of partial discharge current formation in twisted pair.

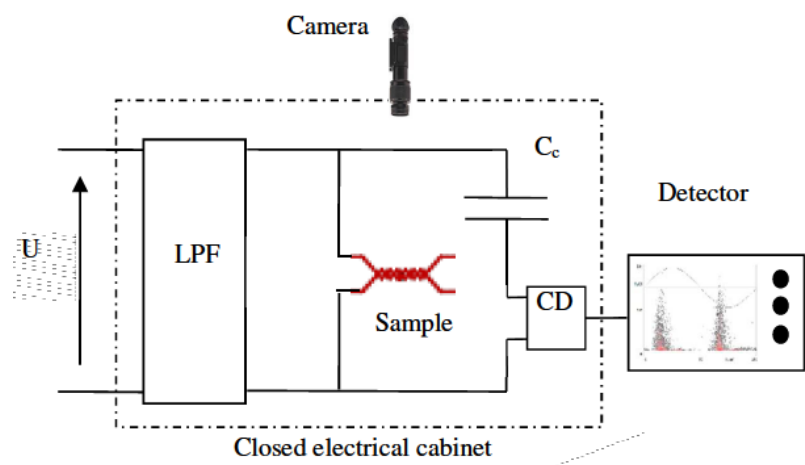

Figure 2: Simplified test bench of the partial discharge detection.

- $\mathrm{C}_{c}$ : coupling capacitor;

- CD: Coupling Device transfers partial discharge signal to the detector;

- Detector: shapes the signal and displays on LCD screen.

- Camera: night vision device.

All these elements are put into a closed cabinet for electrical safety and also to reduce electromagnetic noise. Pictures were made in a deep darkness with a night vision device called "CoronaFinder" [5] placed at $0.8 \mathrm{~m}$ of the twisted pairs. This optical system is able to make a visible image from the UV and IR radiation emitted by PDs.

\section{Effects of an additional semi-conductive layer}

In a motor coil, the outer surfaces of the wires are in contact together. Therefore, an additional semi-conductive external layer creates equipotential surfaces that reduce strongly electric field in the air between the turns. The elec-

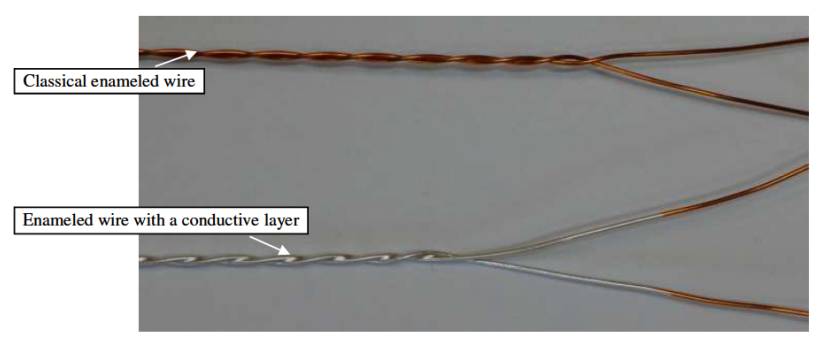

Figure 3: Standard twisted pair and another one covered with a thin layer of silver conductive paint.
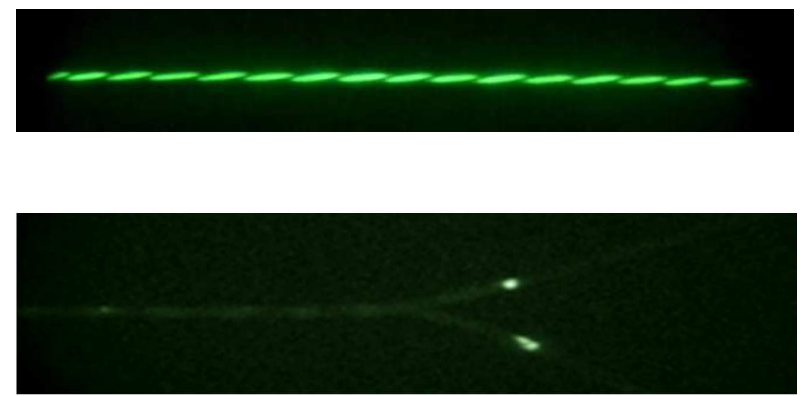

Figure 4: Pictures of twisted pairs in darkness taken by a night vision device at identical voltages (upper picture: standard wire; lower one: modified pair).


Figure 5: PDs pictures in both twisted pairs under identical voltages. Upper picture: standard enameled wire. Lower picture: enameled wire with the external semi-conductive layer.

tric field is concentrated in the inner layers of polymer of the enameled wire insulation, which can withstand high electrical fields [6]. With the thin semi-conductive additional layer, the critical zones, corresponding to high field magnitudes in air, are moved towards the ends of the semiconductive layer.

The first investigations were made on twisted pairs specimens insulated by classical insulation materials. A typical enameled wire is covered of 10 polymer thin layers for avoiding air-voids. The inner ones are made of polyester-imide (PEI) for getting a good adhesion on the copper. The final ones are made of polyamide-imide (PAI), 




(a)

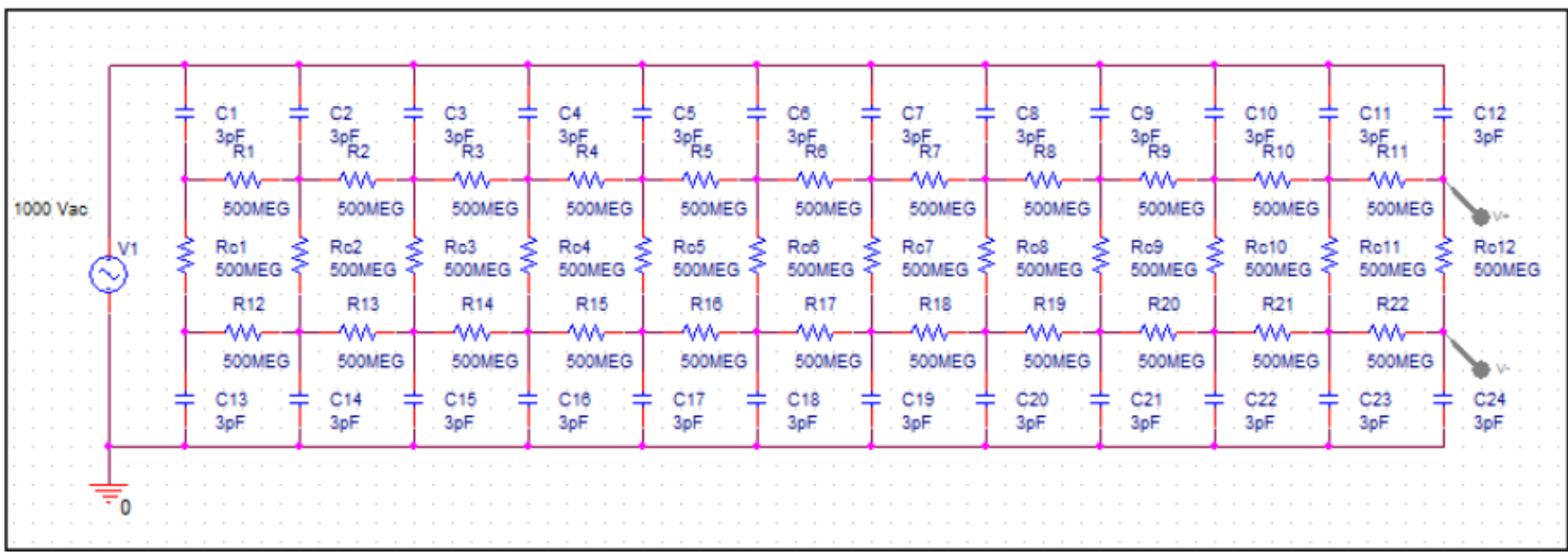

(b)

Figure 6: Equivalent circuit of a twisted pair with semi-conductive external layer.

for getting better thermal properties. Twisted pairs have been prepared according to IEC 60851-5 [7].

The second investigations are achieved with additional conductive layers. On some specimens, a silver conductive paint [8], is added on external layer (Figure 3). The upper sample shows a standard twisted pair and the lower one, a similar twisted pair with an additional silver thin.

A $50 \mathrm{~Hz}$ sine voltage, over the PDIV $\left(1800 V_{\text {peak }}\right)$, feeds the two twisted pair. The upper photography of Figure 4 corresponds to the standard twisted pair; the lower one to the twisted pair covered with the conductive paint. It can be seen that PDs are distributed along the standard twisted pair, but only on the extremities for the modified twisted pair.

\section{Investigations on an enameled wire with a new additional semi-conductive layer}

Obviously, it is impossible to use a conductive paint in a motor winding, because the high electric conductivity of the paint would be equivalent to short circuited turns. The induced current by the variation of the magnetic flux should destroy the winding. However, a better field distribution does not need a high electric conductivity because, at industrial frequencies, the electric field can be considered as static. The influence of the stray capacitances can be neglected. This study highlights the effects of an additional external layer with lower conductivity. It is made of polyamide-imide (PAI) with $2 \%$ of carbon particles. These 
wire samples were used to make standard twisted pairs for taking pictures of PD activity with the same optical device at the same voltage $(1800 \mathrm{~V})$. Figure 5 allows to compare the results: PDs are homogeneously distributed along the twisted pair made with the standard wire but they are not on the second one that uses enameled wire with the external semi-conductive layer. The brightness of the image of PDs is obviously lower. The optical system tuning is the same and the camera pause time also.

The lower picture do not show any concentration of PDs at the ends of the semi-conductive layer as it is observed in tests made with an enameled wire covered by a conducting paint, which has a much higher conductivity (lower part of Figure 4).

\section{Simulation approach}

A numerical model that uses an equivalent circuit of twisted pair made from enamelled wire with semiconductive layer is proposed. It is illustrated in Figure 6 The parameters determination is a very important step for a correct estimation of voltage variations. All the circuit parameter values were measured ( $\mathrm{Ci}$ and $\mathrm{Ri}$ ), except the contact resistance between the surfaces of the wires (boundary of air area, Rci), as this is impossible.

Firstly, the resistance of this additional semiconductive layer is measured by using an experimental sensor device based on the low current measurement method, described by $C$. Lattès at the beginning of the last century [9]. The today version of this sensor is made with an ultra-low input current integrator, the IVC102 [10]. The principle is presented in Figure 7. The external semiconductive layer is used as input resistance. The rectangular input voltage and the triangular output one are presented in Figure 8. The input current is proportional to the slope (dv/dt) of the output voltage. This sensor, calibrated by using a standard resistance, is able to measure currents down to $10 p A$.

Resistance was measured for several lengths of wire. From Figure 9, the resistance is approximated to $25 \mathrm{G} \Omega$ for $50 \mathrm{~cm}$ of wire, so $50 \mathrm{G} \Omega / \mathrm{m}$. Hence, for the numerical model $500 \mathrm{M} \Omega / \mathrm{cm}$ was used as the horizontal resistance Ri.

For getting the equivalent capacitance per centimeter of the twisted pair, the Agilent 4294A precision impedance analyzer is used [11]. The equivalent capacitance of two insulation layers in series is equal to $1.5 \mathrm{pF} / \mathrm{cm}$; thus for the proposed model $\mathrm{Ci}=3 p F$ per insulation.

In order to study the effect of the contact resistance (Rci), simulation starts from $500 M \Omega$, that corresponds to

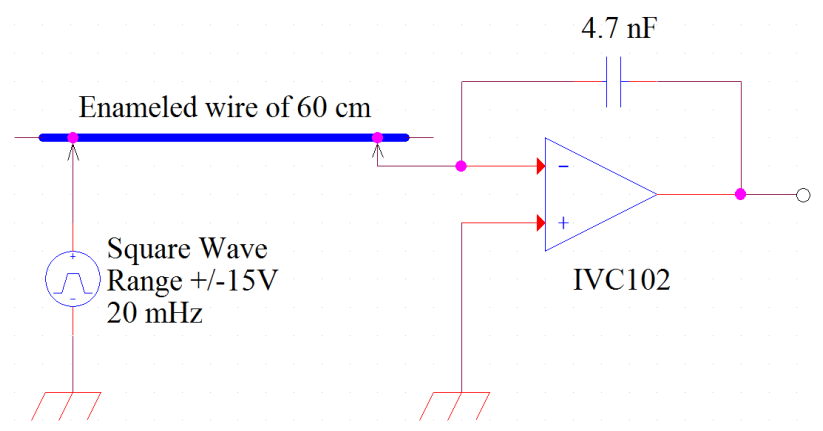

Figure 7: Simplified diagram of the low current measurement.

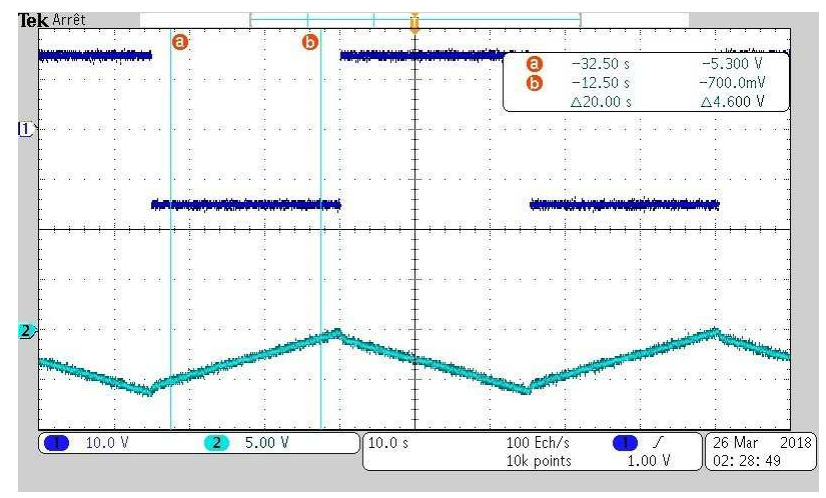

Figure 8: Input (Square) and output (Triangular) voltages of the measuring circuit.

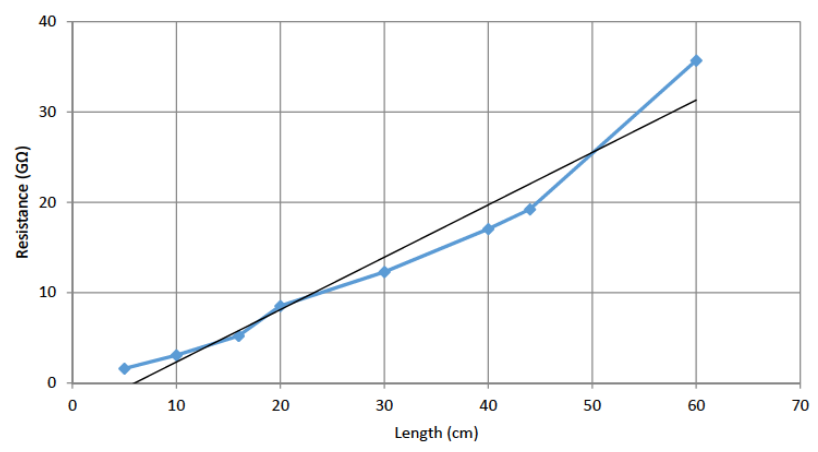

Figure 9: Resistance measurement for several length of the enameled wire with an external semi-conductive layer. Experimental point (blue) and average slope of $500 \mathrm{M} \Omega / \mathrm{cm}$ (black).

the horizontal resistance (of the semi-conductive layer) and then, lower value have been used to see the voltage distribution in air. As it is illustrated on Figure 10, the voltage at the boundary of the air layer decreases with this unknown resistance. Under $50 M \Omega$ the voltage is lower than the PDIV (550V). 


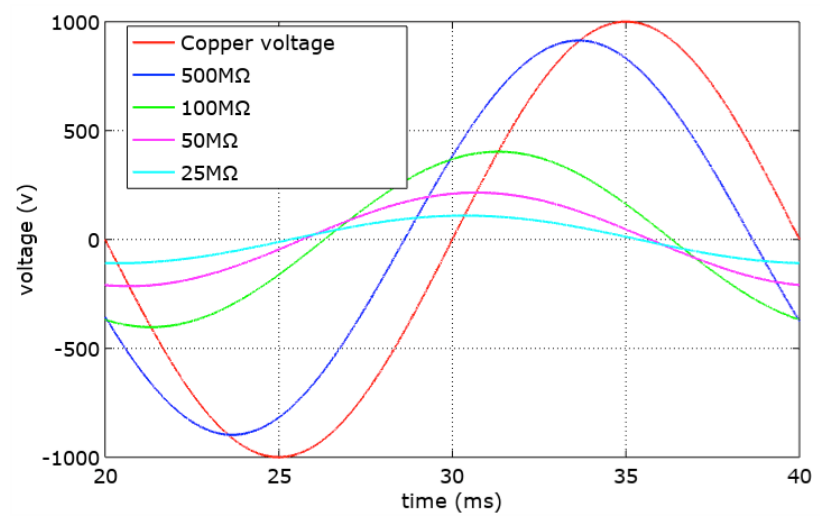

Figure 10: Variation of the voltage depending on the contact resistance between surface wires.

\section{Conclusion}

A new approach for designing ac motor windings free of partial discharges (PDs) is proposed. It consists of adding a thin semi-conductive layer on the outer surface of enameled wires. Thereby, the electrical fields in the residual air-voids existing between turn will be reduced. Two enameled wires with conductive (silver paint) and semiconductive (PAI $+2 \%$ of carbon nanoparticles) external layers have been tested and compared to a classical one. In the first case, PDs occur only at the end of the conductive layer, on the edge of conductive area, rather than randomly, along the turns. Critical zones are well localized. A small additional quantity of varnish in these critical zones, can increase strongly the PDIV. In the second case a mitigation of PD is obtained along the twisted pair, but not the concentration effect on deterministic critical zones. The experimental semiconcuctive layer had a very high specific resistance $(31 \mathrm{G} \Omega / \mathrm{m})$ compared to the silver conducting paint.

Works are in progress for finding an acceptable balance between the PDs concentrations effects that creates a higher PDIV and the additional Joule losses due to the eddy currents in the semi-conductive external layer. Experimental developments must be performed with several kind of conductive particles and different concentrations in order to reduce the value of the contact resistance. This will provide a lower voltage and therefore a reduced electrical stress.

\section{References}

[1] Ye H., Fechner T., Lei Y., Luo T., Zhou M., Han Z., Wang H., Zhuang Q., Xu R., Li D., Review on HVDC cable terminations, High Voltage, IET, 1-30, 2018.

[2] Krpal O., Mraz P., V-A Characteristic Measuring of Stress Grading Tapes in the End-winding of Synchronous Generators, Proc. Engin., Elsevier, 2014, 1523-1528.

[3] Stone G. C., Culbert I., Boulter E. A., Dhirani H., Electrical insulation for rotating machines-design, evaluation, aging, testing, and repair, Wiley-IEEE Press, Hoboken, NJ, 2014.

[4] Standard IEC 60270, High-voltage test techniques. Partial discharge measurements, 3rd ed, 2000.

[5] http://syntronics.net/coronafinder.html.

[6] Roger D., Ait-Amar S., Napieralska E., A method to reduce partial discharges in motor windings fed by PWM inverter, De Gruyter, Open Physics, 2018, Vol 16, No. 1 pp. 599-604.

[7] Standard IEC 60851-5, Winding wires. Test methods. Part 5: Electrical properties, 4th ed, 2008.

[8] https://www.electrolube.com/pdf/tds/044/SCP.pdf

[9] Lattes C., Methode de mesure des faibles courants, Radium (Paris) 6(3), 1909.

[10] http://www.ti.com/lit/ds/symlink/ivc102.pdf.

[11] https://www.upc.edu/sct/ca/documents_equipament/d_271_id658.pdf 